\title{
A Summary of the Experimental Results on Reactions in the Uranium Samples Irradiated with a Deuteron Beam of Energies up to $8 \mathrm{GeV}$ at the QUINTA Target
}

\section{Zavorka ${ }^{1}$}

Czech Technical University, Prague, Czech Republic, Joint Institute for Nuclear Research, Dubna, Russia

E-mail: zavorluk@fjfi.cvut.cz

J. Adam, W. Furman, M. Kadykov, J. Khushvaktov, A. Solnyshkin, V. TsoupkoSitnikov, S.Tyutyunnikov

Joint Institute for Nuclear Research, Dubna, Russia

E-mail: iadam@jinr.ru, furman@dubna.ru,kadykov@jinr.ru, khushvaktov@mail.ru,soln@jinr.ru,vtsoupko@jinr.ru,tsi210647@yandex.ru

\section{P. Caloun}

Nuclear Physics Institute, Rez, Czech Republic

E-mail: caloun@ujf.cas.cz

\section{Chilap}

Center of Physical and Technical Projects “Atomenergomash”, Moscow, Russia

E-mail: chilap@cftp-aem.ru

\section{Suchopar}

Czech Technical University, Prague, Czech Republic,

Nuclear Physics Institute, Rez, Czech Republic

E-mail: martin.suchopar@fjfi.cvut.cz

Natural uranium samples have been irradiated in a neutron field generated via spallation reaction at the QUINTA target (500 kg of ${ }^{\text {nat }} \mathrm{U}$ ). The dependence of the reaction rates of residual nuclei produced inside the samples on the incident deuteron energy of the maximum value of $8 \mathrm{GeV}$ has been investigated. Both the activation measurement technique and the gamma-ray spectrometry have been used. A comparison with MCNPX calculation is enclosed. Nevertheless, the results are still preliminary and also need to be verified by providing further experiments.

XXI International Baldin Seminar on High Energy Physics Problems

September 10-15, 2012

JINR, Dubna, Russia

1

Speaker 


\section{Introduction}

In the early 1990s, the energy amplifier [1], a basic concept of a device for extracting nuclear energy with the use of particle beam accelerator induced nuclear reactions, was introduced. An idea of such an apparatus was further developed and presented in Ref. [2]. At that time, a thought of Accelerator-driven systems (ADS) started to represent an attractive alternative for future safe, environment-friendly and affordable nuclear energy systems.

The main characteristic features of ADS can be summarized as follows: a neutron production is provided by the spallation reaction in a heavy-element target (e.g. $\mathrm{W}, \mathrm{Pb}, \mathrm{Th}, \mathrm{U}$ ). Contrary to the currently operating nuclear reactors, the above mentioned aspect can assure ADS operation under the subcritical conditions in a closed fuel cycle. Moreover, considering the fast neutron spectrum in ADS [3], these systems can be effectively used for transmutation of both the long-lived fission fragments and the transuranic elements originated in the current nuclear power plants and thus to eliminate a not negligible amount of nuclear waste destined for geological repositories.

At the present time a design of ADS-based systems is being developed in many scientific laboratories all over the world, e.g. TEF at J-PARC, Japan [4], MYRRHA reactor at SCK·CEN in Belgium [5] or ADS programme at BARC, India [6]. Relativistic Nuclear Technology (RNT) represents a specific project of the deep subcritical electronuclear systems. The RNT project has been designed by the certain members of the international collaboration "E\&T-RAW". Currently, the project is being investigated at the Joint Institute for Nuclear Research (JINR), Dubna, Russia, with the use of Nuclotron accelerator as a basic experimental equipment $[7,8]$.

The RNT design is based mainly on the principle of an increase in energy of incident beam particles up to $10 \mathrm{GeV}$ in comparison with the value of $1 \mathrm{GeV}$ regarding the traditional ADS. As demonstrated in Ref. [9], with the increasing beam energy also grows the hardness of neutron spectra in the spallation target. In addition to the extensive neutron multiplicity, this aspect represents a crucial precondition for the successful and efficient course of both the highenergy fission and the transmutation reaction in a quasi-infinite multiplying system.

For this reason a scientific interest of the whole "E\&T-RAW" collaboration is currently focused on a physics research on the massive natural uranium target QUINTA. The fast neutron spectrum inside the target can be achieved through the spallation reaction using the deuteron beam having the maximum available energy $E_{\mathrm{d}}=8 \mathrm{GeV}$.

A basic physics research concerning the RNT includes, among others, the investigation of the main reaction processes occurring in natural uranium, the determination of generated products and corresponding reaction rates. It is also worth comparing the experimental results with the Monte Carlo calculations provided by MCNPX code [10].

For this purpose some natural uranium samples were installed into certain position inside the QUINTA target during the $43^{\text {rd }}$ and the $45^{\text {th }}$ Nuclotron run in 2011 and 2012. Recent results are presented in this paper. 


\section{Experimental conditions}

\subsection{The QUINTA target}

The QUINTA target [11] represents the last commissioned experimental setup of the "E\&T-RAW" collaboration. The setup is composed of about $500 \mathrm{~kg}$ of natural uranium. The schematic drawings illustrating the whole target assembly and a location of natural uranium samples inside the target can be observed in Fig. 1a and Fig. 1b.

The target consists of five sections separated by an air gap. Each section is $114 \mathrm{~mm}$ long; the value of $350 \mathrm{~mm}$ represents both the width and height of any hexagonal shaped section. The first section facing the deuteron beam includes 54 uranium rods and the central beam window of $80 \mathrm{~mm}$ in diameter. The uranium rods are characterized by the length of $104 \mathrm{~mm}$ and the diameter of $36 \mathrm{~mm}$. Due to safety requirements, every rod has to be hermetically sealed in an aluminum shell. Additionally, each of the target units is also situated in the aluminum casing. Excluding the first section, the other sections are composed of 61 uranium rods. The target as a whole is nearly $700 \mathrm{~mm}$ long and of $540 \mathrm{~kg}$ in weight including all construction materials. The biological shielding is built of lead bricks.

Investigated experimental samples are usually installed at the sample holders. The holder can easily be inserted into, resp. removed from the air gap between the selected target sections. Thus, the sample can be irradiated in different positions depending both on radius ( $y$ axis) and length ( $z$ axis) of the target.

Finally, the target assembly is situated on the movable platform ensuring nearly effortless motion in the Nuclotron experimental hall. Before beginning the irradiation, the experimental setup needs to be precisely installed in the way that the deuteron beam could hit the center of the beam window.

a)

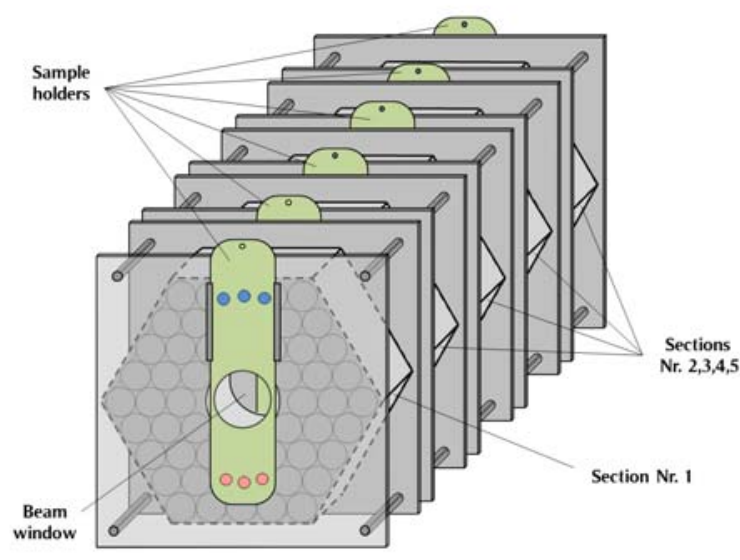

b)

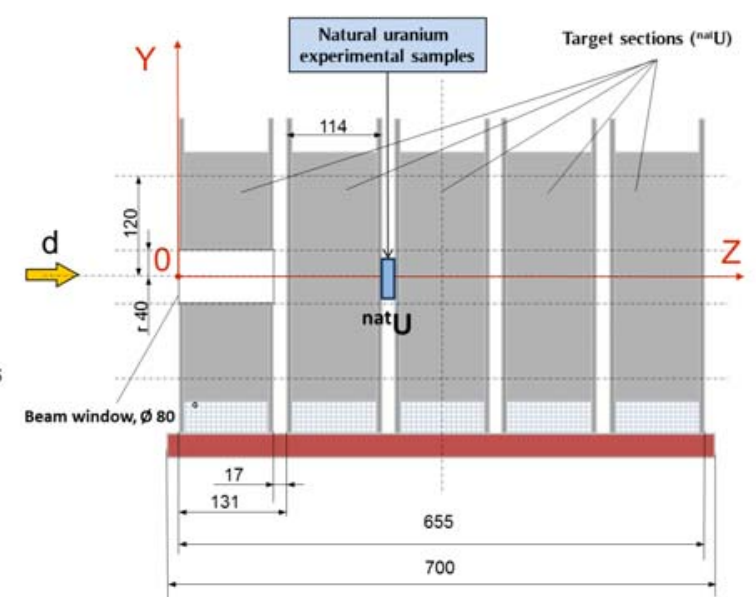

Fig. 1. Schematic drawings of the QUINTA target: (a) general view of the target and (b) the position of investigated natural uranium samples (sample not to scale) 


\subsection{Natural uranium samples}

As mentioned above, the natural uranium samples were installed at the QUINTA target in order to investigate the basic characteristics of the reactions occurring inside the uranium target assembly. The cylindrical shaped samples of several tenths gram in weight were situated at the central radial position $(y=0)$ between the second and the third section of the QUINTA target (see Fig. 1b.). As follows from previous related experiments provided by "E\&T-RAW" collaboration, there should be expected the maximum neutron flux in this position.

As commonly known, natural uranium is mainly composed of ${ }^{235} \mathrm{U}$ and ${ }^{238} \mathrm{U}$ isotope of natural abundance $0.72 \%$, resp. $99.27 \%$. Whereas the former can easily be split by thermal neutrons because of a high cross-section value ( $1 / v$ law in the low energy region), the energy threshold for fission of the latter can not be found until reaching the region of about $1 \mathrm{MeV}$. Thus, in order to estimate an impact of thermal neutrons, some uranium samples were covered by the thin (approx. $2 \mathrm{~mm}$ ) layer of cadmium serving as a thermal neutron shielding. Since the main emphasis of the experiment is put on fast neutrons, an influence of thermal neutrons on the total number of fission reactions in the ${ }^{\text {nat }} \mathrm{U}$ samples can reliably be determined in this manner.

\subsection{The irradiation process}

The uranium target was irradiated with the deuteron beam of JINR Nuclotron. A range of beam energies covered the interval from $1 \mathrm{GeV}$ up to $8 \mathrm{GeV}$, namely $1,2,4,6$ and $8 \mathrm{GeV}$. The total number of deuterons hitting the target reached at least the order of $10^{12}$ particles per one experimental run (the lowest figure is represented by the value of $(5.39 \pm 0.17 \pm 0.54) \cdot 10^{12}$ deuterons in case of $8 \mathrm{GeV}$ run; the highest value of $(2.72 \pm 0.07 \pm 0.27) \cdot 10^{13}$ deuterons was reached during the $4 \mathrm{GeV}$ experiment). The maximum time duration of the irradiation process was no longer than 20 hours.

The total beam intensity was determined with the use gamma-ray spectrometry. Several thin aluminum foils were located between the beam output of the accelerator and the beam window of the QUINTA target. The ${ }^{27} \mathrm{Al}(\mathrm{d}, \mathrm{x})^{24} \mathrm{Na}$ beam monitor reaction was used. The reaction cross-section values were obtained by fitting the data taken from Ref. [12, 13]. The resulting values regarding the total beam intensity are listed in Ref. [14]. An uncertainty in the crosssection evaluation was there established to be $10 \%$.

In order to exclude an influence of back scattered neutrons on the beam intensity determination via the ${ }^{27} \mathrm{Al}(\mathrm{n}, \alpha)^{24} \mathrm{Na}$ reaction, one of $\mathrm{Al}$ foils was situated directly at the beam output, at a distance of at least $2.5 \mathrm{~m}$ from the target.

The ionization chamber was used for monitoring of the beam intensity time profile during all irradiation processes. The beam intensity record regarding the $1 \mathrm{GeV}$ experiment is presented in Fig. 2a. A corresponding beam intensity fluctuation correction factor can be found in Fig. 2b. This spectroscopy correction factor is necessary to take into consideration while processing the data of radioactive isotopes depending on their half-lives. It seems to be essential for further data analysis, especially in case of short-lived residual nuclei. 
a)

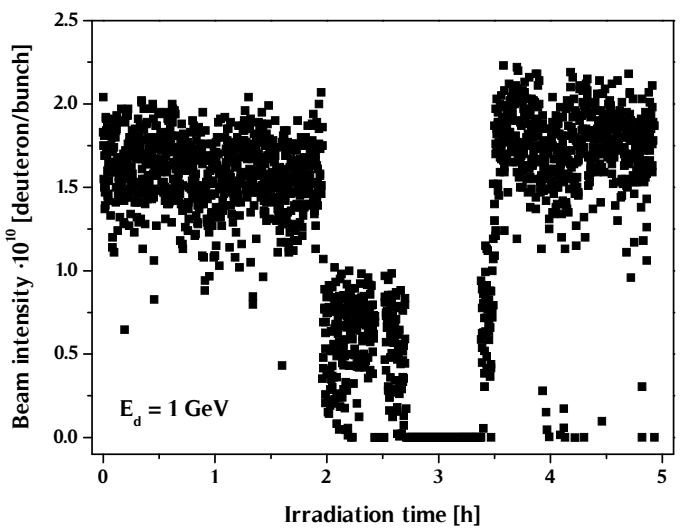

b)

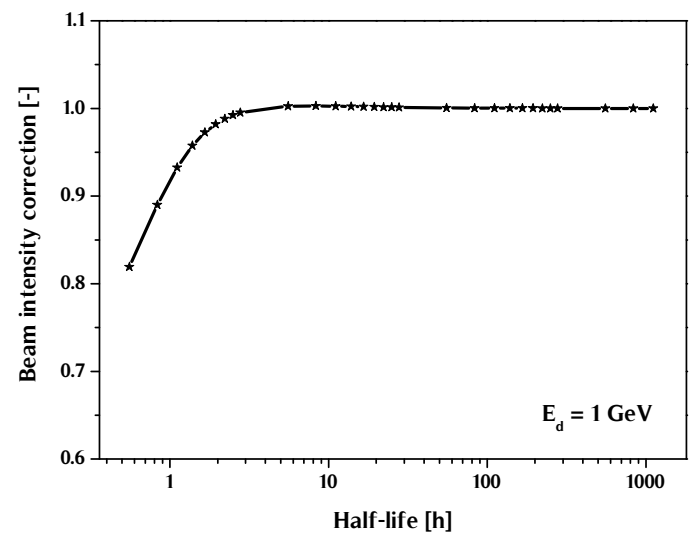

Fig. 2. Deuteron beam characteristics of the $1 \mathrm{GeV}$ irradiation (a) beam intensity record and (b) beam intensity fluctuation correction factor depending on the half-life of residual nuclei

\section{Measurement methods}

After finishing the irradiation, the gamma-ray spectrometry was used in order to reach experimental data on reactions in the uranium samples. Properly shielded and well calibrated HPGe detectors with a relative efficiency of $18 \%$ and $32.9 \%$ were utilized during all the measurements.

For the purpose of an appropriate data acquisition concerning the residual nuclei with a short half-life, a set of measurements started soon after the termination of each irradiation. Subsequently, for receiving enough data regarding the long-lived isotopes, measurements have been continuing for a long period after the end of irradiation. The time duration of measurements gradually rose up from 10 minutes up to 5 days. It is worth mentioning that each of the uranium samples was measured at least ten times.

The measured uranium gamma-ray spectra were processed using the DEIMOS code [15]. An identification procedure of residual nuclei was performed on the basis of characteristic gamma-line energy, intensity and corresponding half-life taken from Ref. [16].

\section{Data analysis}

For further analysis it is convenient to arrange the data in the form of reaction rate $R\left(A_{r}, Z_{r}\right)$ of the specific isotope characterized by mass number $A_{r}$ and atomic number $Z_{r}$. The reaction rate as mentioned in Ref. [17] is defined as the number of produced residual nuclei $Q\left(A_{r}, Z_{r}\right)$ per number of atoms $N_{\mathrm{t}}$ in the sample per incident deuteron per second $N_{\mathrm{d}}$ as follows:

$$
R\left(A_{r}, Z_{r}\right)=\frac{Q\left(A_{r}, Z_{r}\right)}{N_{\mathrm{t}} \cdot N_{\mathrm{d}}} .
$$


Taking the reaction rate into account, it is then not exacting to present the results in the form related e.g. per gram of the sample (as presented later in this paper), per total number of deuterons etc.

The number of produced residual nuclei $Q\left(A_{r}, Z_{r}\right)$ follows from the formula mentioned in Ref. [18]. This represents a pure gamma-spectrometry quantity. It depends on the corresponding peak area, energy and intensity of gamma-line, half-life of radioactive nucleus, duration of measurement, cooling time and includes some spectroscopy corrections as well.

Excluding the beam intensity fluctuation correction factor (see par. 2.3), the total correction factor also includes the detector efficiency correction, correction on dead time, selfabsorption correction coefficient and correction on decay during irradiation, cooling and measurement.

\section{MCNPX calculation}

A Monte Carlo simulation was carried out in order to compare experimental and calculated values of reaction rates. The reaction rate calculation for the respective residual nucleus can be performed in the following way:

$$
R\left(A_{r}, Z_{r}\right)=\int_{E_{\text {th }}}^{E_{\max }} \sigma\left(A_{r}, Z_{r}, E_{n}\right) \cdot \phi\left(E_{n}\right) d E_{n},
$$

where $E_{\mathrm{th}}$, resp. $E_{\max }$ denote threshold and maximum energy of the reaction, $\sigma$ has a meaning of the reaction cross-section and $\phi$ represents neutron flux depending on the respective neutron energy $E_{n}$.

The simulation was performed with the use of the MCNPX 2.7a code. The complete geometric model of the target set-up was prepared [19] as a part of the input file. The neutron flux simulations were implemented combining the INCL4 intranuclear physics model and the ABLA fission-evaporation model.

Furthermore, for the purpose of the MCNPX input file, the deuteron beam profile (i.e. horizontal and vertical value of FWHM and shift of the Gaussian beam profile) was experimentally detected by solid state track detectors [20].

Whereas the results on the neutron flux in Eq. (2) can be obtained using the MCNPX code, the corresponding reaction cross-section values need to be calculated by the NJOY [21] code and TALYS-1.4 computer program [22].

\section{Results and discussions}

By analyzing the gamma-ray spectra of ${ }^{\text {nat }} U$ samples, more than one hundred residual nuclei have been reliably identified. A wide presence of many different products of (n, $\gamma),(n, 2 n)$ and $(\mathrm{n}, \mathrm{f})$ reactions was confirmed during the analysis. Some results can be already presented in this paper. 


\subsection{Fission reaction}

As mentioned in section 2.2, some uranium samples were covered by cadmium shielding in order to determine a contribution of thermal neutrons to the total number of fission events. No significant contribution was expected with regard to the fast neutron spectrum field in the whole uranium target. The expectation has been experimentally confirmed within the statistical uncertainties since the value 1.05(12) represents the ratio of reaction rates for the fission of nonshielded and Cd-covered ${ }^{\text {nat }} \mathrm{U}$ samples during $1 \mathrm{GeV}$ irradiation. Regarding this case, there are presented the experimental values of reaction rates of identified residual nuclei in Fig. 3a.

Tab. 1. The main characteristics of ${ }^{99} \mathrm{Mo},{ }^{131} \mathrm{I},{ }^{133} \mathrm{I}$ and ${ }^{143} \mathrm{Ce}$ isotopes $\left(I_{\gamma}>10 \%\right.$ listed)

\begin{tabular}{llll} 
& Half-life $\mathrm{T}_{1 / 2}$ & Energy $\mathrm{E}_{\gamma}[\mathrm{keV}]$ & Intensity $\mathrm{I}_{\gamma}[\%]$ \\
\hline${ }^{99} \mathrm{Mo}$ & $65.94(1) \mathrm{h}$ & $140.511(1)$ & $89.43(23)$ \\
& & $739.50(2)$ & $12.13(12)$ \\
\hline${ }^{131} \mathrm{I}$ & $8.0207(1) \mathrm{d}$ & $364.489(5)$ & $81.7(6)$ \\
\hline${ }^{133} \mathrm{I}$ & $20.8(1) \mathrm{h}$ & $529.872(11)$ & $87.0(17)$ \\
\hline${ }^{143} \mathrm{Ce}$ & $33.039(6) \mathrm{h}$ & $293.266(2)$ & $42.80(13)$
\end{tabular}

a)

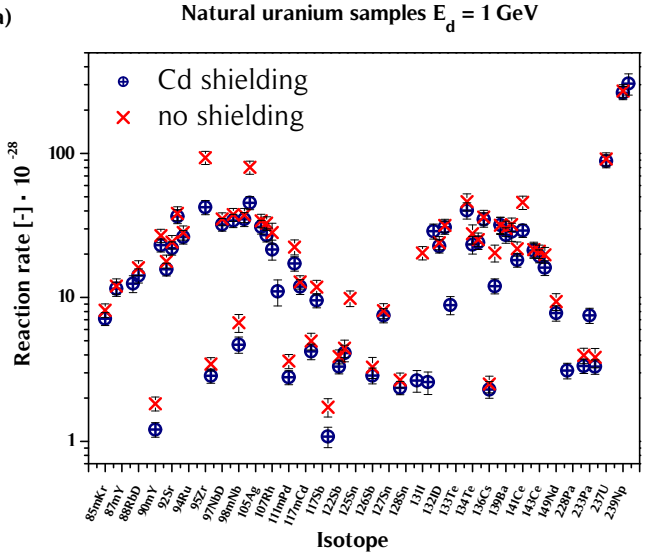

b)

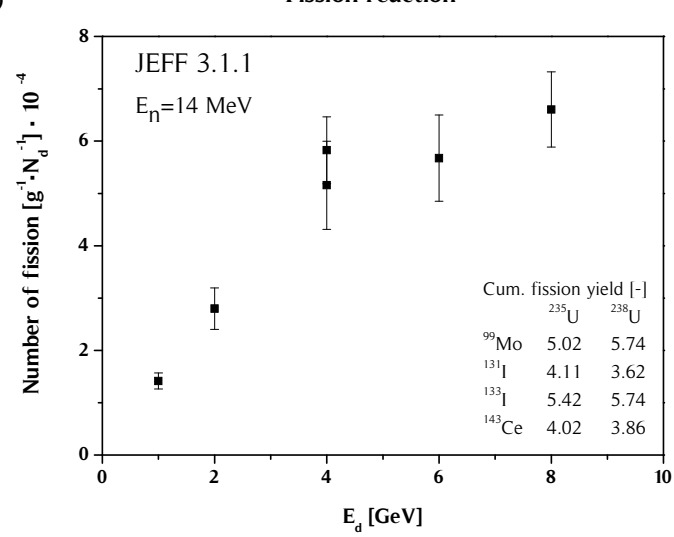

Fig. 3. Preliminary experimental results on reactions in ${ }^{\text {nat }} U$ samples: (a) a comparison of reaction rates for non-shielded and Cd-covered samples (not all isotopes mentioned in legend) and (b) the number of fission according to ${ }^{99} \mathrm{Mo},{ }^{131} \mathrm{I},{ }^{133} \mathrm{I}$ and ${ }^{143} \mathrm{Ce}$ isotope production considering JEFF 3.1.1, $E_{\mathrm{n}}=14 \mathrm{MeV}$ cumulative fission yield library

Since the fast neutron fission seems to be the main reaction mechanism occurring inside the samples, it is then possible to assess the dependence of the total number of fission in ${ }^{\text {nat }} \mathrm{U}$ on the incident deuteron beam energy. The isotopes mentioned in Tab. 1. were selected in order to determine the total number of fission events. A selection of these isotopes was based on suitable values of half-life, energy and intensity of gamma-ray lines. Moreover, the selected isotopes need to be characterized by an adequate fission yield value. 
The cumulative fission yields for the fission of ${ }^{235} U$ and ${ }^{238} U$ there were taken from Ref. [23] for the maximum available energy of neutrons $E_{\mathrm{n}}=14 \mathrm{MeV}$. Proportionally to the natural uranium abundance, the cumulative fission yields were assigned to the experimental reaction rate values of the corresponding residual nuclei. Weighted average of the total number of fission events in ${ }^{\text {nat }} \mathrm{U}$ samples is presented in Fig. 3 b.

A pronounced increase in the number of fission in dependence on the incident deuteron energy can be taken into consideration as an important result of this experimental study.

Nevertheless, some isotopes, e.g. ${ }^{82} \mathrm{Br},{ }^{122} \mathrm{Sb},{ }^{136} \mathrm{Cs}$ have been produced in such amount which does not correspond to the expected values according to [23] (just for illustration purpose: ${ }^{82} \mathrm{Br}$ was produced $(5.0 \pm 1.0) \cdot 10^{3}$ times more, ${ }^{122} \mathrm{Sb}$ isotope $(1.3 \pm 0.2) \cdot 10^{5}$ times and ${ }^{136} \mathrm{Cs}$ $(6.2 \pm 0.7) \cdot 10^{1}$ times more than expected in the case of $8 \mathrm{GeV}$ run). It means that the fast neutron fission is the main but probably not the only one reaction mechanism taking place in the issue.

\subsection{Experimental results vs. simulation}

In accordance with Eq. (2), in order to obtain calculated values of reaction rates, a computation of the reaction cross-section and neutron flux is required. The cross-section values were obtained using the NJOY code up to the maximum neutron energy $E_{n}=20 \mathrm{MeV}$. Between this value and $E_{n}=200 \mathrm{MeV}$, the TALYS program was used. Finally, above $200 \mathrm{MeV}$, the cross-section values were obtained by extrapolating.

The following reactions were taken into account for the purpose of comparison: ${ }^{238} U(n, \gamma){ }^{239} U,{ }^{238} U(n, 2 n){ }^{237} U$, and ${ }^{n a t} U(n, f) F P$. The product of the former can be experimentally detected in an undemanding manner through its decay daughter product, ${ }^{239} \mathrm{~Np}$ (convenient halflife, energy and intensity of gamma-ray lines); ${ }^{237} \mathrm{U}$ isotope can be successfully detected directly and fission products (FP) represent a wide range of values of spectrometry quantities. In Tab. 2, there are presented the currently available results on the comparison of the experimental and the calculated values of reaction rates (i.e. $E_{d}=2$, 4 , and $6 \mathrm{GeV}$ regarding the $43^{\text {rd }}$ Nuclotron run).

\section{Conclusions}

A brief introduction of the massive uranium target QUINTA as a promising tool for experimental investigation of ADS-based systems was presented in this paper. The natural uranium samples inside the target were subjected to research in a set of extensive experiments.

Tab. 2. The comparison of experimental and calculated production reaction rates concerning the experiments of 2, 4, and $6 \mathrm{GeV}$ deuteron beam energy; preliminary results.

\begin{tabular}{llcc} 
Reaction & \multicolumn{3}{l}{$\begin{array}{l}\mathrm{E}_{\mathrm{d}}=2 \mathrm{GeV} \\
\text { Ratio of experimental / calculated reaction rates }\end{array}$} \\
\hline${ }^{238} \mathrm{U}(\mathrm{n}, \gamma)^{239} \mathrm{U}$ & $1.22(13)$ & $2.43(25)$ & $1.94(20)$ \\
\hline${ }^{238} \mathrm{U}(\mathrm{n}, 2 \mathrm{n}){ }^{237} \mathrm{U}$ & $1.01(10)$ & $2.24(24)$ & $1.53(16)$ \\
\hline${ }^{\text {nat }} \mathrm{U}$ fission & $0.93(10)$ & $2.19(23)$ & $1.46(16)$
\end{tabular}


Analyzing measured gamma-ray spectra it was demonstrated that thermal neutrons do not play any significant role in the fission of investigated ${ }^{\text {nat }} U$ samples. As presented in Fig. 3a, the reaction rates of produced residual nuclei indicate two extremes. One maximum can be found in the region of mass number $A_{r}=95$, the other is situated around the value of 135 .

In Fig. 3b, there can be seen how considerably the total number of fission events in ${ }^{\text {nat }} \mathrm{U}$ increases in dependence on the incident deuteron beam energy.

Furthermore, as follows from the comparison of the experimental and the calculated values of the reaction rate for ${ }^{238} \mathrm{U}(\mathrm{n}, \gamma){ }^{239} \mathrm{U},{ }^{238} \mathrm{U}(\mathrm{n}, 2 \mathrm{n}){ }^{237} \mathrm{U}$, and ${ }^{\text {nat }} \mathrm{U}(\mathrm{n}, \mathrm{f}) \mathrm{FP}$ reactions presented in Tab. 2., a relatively good agreement in the case of $2 \mathrm{GeV}$ experiment is not so evident considering 4 and $6 \mathrm{GeV}$ irradiation. The disagreement can be caused either by an inaccurate MCNPX physics model in the region of higher energies or by an imperfect cross section evaluation. A sensitivity of performed calculations for the deuteron beam shape and position determination can play a significant role as well. Naturally, the results on the experimental data can also be considerably responsible for the disagreement.

Since the measured gamma-ray spectra are very extensive and the data processing rather complicated process, all the data need an additional analysis provided in more detail. Finally, complementary results considering the additional values of the incident deuteron beam energy should be completed by providing further experiments.

\section{References}

[1] F. Carminati et al., An energy amplifier for cleaner and inexhaustible nuclear energy production driven by a particle beam accelerator, CERN Preprint, CERN/ AT / 94-47 (ET), 1993

[2] C. Rubbia et al., Conceptual design of a fast neutron operated high power energy amplifier, CERN Preprint, CERN/ AT / 95-44 (ET), 1995

[3] A. Herrera-Martínez et al., Transmutation of nuclear waste in accelerator-driven systems: Fast spectrum, Annals of Nuclear Energy 34 (2007) 564-578

[4] S. Tanaka, High intensity proton accelerator project in Japan (J-PARC), Radiation Protection Dosimetry 115 (2005) 33-43

[5] H.A. Abderrahim et al., MYRRHA, a Multipurpose hYbrid Research Reactor for High-end Applications, Nucl. Phys. News, Vol. 20, No. 1, 2010

[6] P. Singh et al., Accelerator development in India for ADS programme, Pranama - J.Phys., Vol. 68, No. 2, 2007, 331-342

[7] «E\&T RAW» Collaboration, Study of deep subcritical electronuclear systems and feasibility of their application for energy production and radioactive waste transmutation, JINR Preprint, E1-2010-61, Dubna, 2010

[8] A. Baldin et al., Relativistic Nuclear Technology (RNT) for energy production and utilization of spent nuclear fuel. The results of first experiments on physical justification of RNT, JINR Preprint, E1-2011-24, Dubna, 2011 
[9] V. Yurevich, Production of neutrons in thick targets by high-energy protons and nuclei, Physics of Particles and Nuclei 41 (2010) 778-825

[10] D. Pelowitz, MCNPX ${ }^{T M}$ User's manual, Version 2.7.0, LA-CP-11-00438, 2011

[11] M. Kadykov et al., About the possibilities of the ADS, based on the use of natural uranium or thorium for energy production and processing of nuclear waste, $13^{\text {th }}$ Int. Sci. Conf. Electric Power Engineering, 23.-25.5.2012, Brno, Czech Republic

[12] J. Banaigs et al., Determination de l'intensite d'un faisceau de deutons extrait d'un synchrotron et mesure des sections efficaces des reactions ${ }^{12} \mathrm{C}(d, p 2 n)^{11} \mathrm{C}$ et ${ }^{27} \mathrm{Al}(\mathrm{d}, 3 p 2 n)^{24} \mathrm{Na}$ à $2.33 \mathrm{GeV}$, Nucl. Instr. and Meth. B 95 (1971) 307-311

[13] P. Kozma et al., Application of $\mathrm{BaF}_{2}$ scintillator to off-line gamma-ray spectroscopy, Czechoslovak Journal of Physics, 40 (1990) 393-397

[14] V. Wagner, The intensity of the deuteron beams on Kvinta set-up during December 2011 experimental campaign, "E\&T-RAW" collaboration report, 2012

[15] J. Frána, Program DEIMOS32 for gamma-ray spectra evaluation, J. Radioanal. Nucl. Chem. 257 (2003) 583-587

[16] S.Y.F. Chu, L.P. Ekström and R.B. Firestone, WWW Table of Radioactive Isotopes, database version 2/28/1999 from URL http://nucleardata.nuclear.lu.se/nucleardata/toi/

[17] J. Adam et al., A study of reaction rates of $(n, f),(n, g)$ and $(n, 2 n)$ reactions in ${ }^{n a t} U$ and ${ }^{232}$ Th by the neutron fluence produced in the graphite set-up (GAMMA-3) irradiated by $2.33 \mathrm{GeV}$ deuteron beam, Eur. Phys. J. A (2011) 47: 85

[18] J. Adam et al., A study of nuclear transmutation of Th and ${ }^{\text {nat }} \mathrm{U}$ with neutrons produced in $\mathrm{Pb}$ target and U blanket irradiated by 1.6 GeV deuterons, Eur. Phys. J. A 43 (2010) 159-173

[19] M. Suchpár, Monte Carlo Simulations of Natural Uranium Setups Irradiated With Relativistic Deuterons by Means of MCNPX Code, XXI International Baldin Seminar on High Energy Physics Problems, JINR Dubna, Russia, September 10-15, 2012

[20] I. Zhuk et al., Deuterons beam parameters measurements of the Nuclotron using SSNTD, XXI International Baldin Seminar on High Energy Physics Problems, JINR Dubna, Russia, September 10-15, 2012

[21] Oak Ridge National Laboratory, RSICC Peripheral shielding routine collection NJOY99.0, ORNL, PSR-480, NJOY99.0, 2000

[22] A. Koning et al., TALYS-1.4 A nuclear reaction program User manual, Nuclear Research and Consultancy Group (NRG), The Netherlands, 2011

[23] A. Santamarina et al., The JEFF-3.1.1 Nuclear Data Library, JEFF Report 22, Validation Results from JEF-2.2 to JEFF-3.1.1, OECD, NEA No. 6807, ISBN 978-92-64-99074-6, (2009) 